\title{
Perfil hematológico de búfalas da raça Murrah, criadas ao sol e à sombra, em clima tropical da Amazônia Oriental
}

\author{
Jamile Andréa Rodrigues SILVA¹, Airton Alencar ARAÚJO², José de Brito Lourenço JÚNIOR³ \\ Batista VIANA ${ }^{4}$, Nùbia de Fátima Alves SANTOS ${ }^{5}$, Alexandre Rossetto GARCIA ${ }^{6}$
}

\begin{abstract}
RESUMO
Foram avaliadas as respostas hematológicas de 20 búfalas, criadas ao sol (grupo - SS) e à sombra (grupo - CS), em Belém, Pará. Os animais do grupo CS ( $\mathrm{n}=10)$ estavam em sistema silvipastoril, com Racosperma mangium e os do SS ( $\mathrm{n}=10)$, em piquetes sem sombra, em pastagem de Brachiaria humidicola, água para beber e sal mineral. Foram mensuradas temperatura do ar (TA), umidade relativa do ar (UR) e temperatura de globo negro, em cada tratamento. A coleta de sangue para eritrograma e número total de leucócitos foi realizada a cada 14 dias, às 13 h, durante o ano de 2009. Através da análise de variância constatou-se que em todos os períodos do ano, a TA e índice de temperatura de globo e umidade (ITGU) foram diferentes $(\mathrm{P}<0,05)$, com níveis mais elevados no grupo SS. No período mais chuvoso, o grupo CS apresentou valores elevados de leucócitos, enquanto nos períodos de transição e menos chuvoso, foram maiores no grupo SS. No período menos chuvoso do ano, o grupo SS apresentou maiores valores de hemácias. $\mathrm{O}$ teor de hemoglobina teve maiores níveis $(\mathrm{P}<0,05)$ nos períodos de transição e menos chuvoso. Somente a hemoglobina teve correlaçáo significativa e negativa $(\mathrm{P}<0,05)$ com a UR. Conclui-se que as novilhas búfalas Murrah estão sujeitas a um ambiente hostil e o período menos chuvoso é o mais propício a causar estresse térmico.
\end{abstract}

PALAVRAS-CHAVE: hematologia, búfalos, bioclimatologia.

\section{Hematologic profile of murrah buffalo cows, raised in the sun and under shade, at tropical climate of the eastern amazon}

\section{ABSTRACT}

We evaluated the hematologic responses of 20 female buffaloes raised under full sun (group NS) and shade (group SG) at Belem, Para, Brazil. The animals in the SG group $(n=10)$ were maintained in paddocks under a silvopastoral system with Racosperma mangium, and the ones in the NS ( $\mathrm{n}=10)$, in paddocks with Brachiaria bumidicola, with drinking water and mineral salt, but without access to shade. Air temperature (AT), relative air humidity (RH) and black global temperature (BGT) were measured for each treatment. Blood sampling for erythrogram and leukocyte count were undertaken at intervals of 14 days, and carried out at 1:00 p.m., during 2009. AT and black global temperature and humidity index (BGHI) were different (P< $0.05)$ throughout the year, and their highest values were observed in the NS group. During the rainier period, the SG group presented the highest values of leukocytes $(\mathrm{P}<0.05)$, although in the transition and low rainy periods, the highest values were observed in the NS group. In the low rainy period, the erythrocytes count showed the highest values in the NS group. The highest levels of hemoglobin $(\mathrm{P}<0.05)$ were observed during the transition and low rainy periods. Only hemoglobin presented a significant and negative $(\mathrm{P}<0.05)$ correlation with $\mathrm{RH}$. We concluded that the female Murrah buffaloes are subject to a hostile environment, and that the low rainy period is the most favorable for causing heat stress.

KEYWORDS: hematology, buffaloes, bioclimatology.

\footnotetext{
1 Universidade Federal Rural da Amazônia. jamileandrea@yahoo.com.br

2 Universidade Estadual do Ceará. aaalencar2002@yahoo.com.br

3 Universidade Federal do Pará e Universidade Federal Rural da Amazônia. joselourencojr@yahoo.com.br

${ }^{4}$ Universidade Federal Rural da Amazônia. rinaldo.viana@ufra.edu.br

${ }^{5}$ Universidade Federal Rural da Amazônia. nubiasaint@yahoo.com.br

${ }^{6}$ Embrapa Amazônia Oriental. argarcia@cpatu.embrapa.br
} 
Perfil hematológico de búfalas da raça Murrah, criadas ao sol e à sombra, em clima tropical da Amazônia Oriental

\section{INTRODUÇÃO}

O clima da Amazônia Oriental é quente e úmido, com temperatura do ar média anual de $27^{\circ} \mathrm{C}$, umidade relativa do ar de $85 \%$ e precipitação pluvial de $3.001,3 \mathrm{~mm}$ (Bastos et al. 2002). Nessas condiçôes climáticas, a criação de búfalos se desenvolve de maneira satisfatória, nos diferentes ecossistemas de pastagens nativas e cultivadas na terra firme e várzeas, propícias aos bubalinos. Por outro lado, quando expostos a elevadas temperaturas e sob exposição à radiação solar direta, há decréscimo no desempenho produtivo e reprodutivo. Assim, a adoção de sistemas de produçáo, como os silvipastoris (SSP), são de grande importância, uma vez que o uso das árvores na pastagem diminui a intensidade da radiação solar direta, proporcionando maior conforto térmico e, consequentemente, melhor produtividade dos bubalinos (Lourenço Júnior et al. 2006).

Como indicadores de estresse térmico, os parâmetros hematológicos têm sido utilizados mundialmente (Paes et al. 2000). Entretanto, vários fatores devem ser considerados, tais como raça, espécie, idade, sexo, hora do dia e estado fisiológico, uma vez que podem interferir nos valores de referência para sua interpretação (Jain 1993). Assim, sem uma avaliação adequada, valores hematológicos obtidos para animais criados em determinada regiáo não devem ser considerados de referência para outra (Birgel Júnior et al. 2001).

Portanto, podem ocorrer variações hematimétricas, em condiçóes estressantes, o que modifica a morfologia e a composição celular (Barcelos et al. 1989). Considerando-se que são praticamente inexistentes informações hematológicas relacionadas ao estresse térmico de bubalinos na Amazônia, objetivou-se, com este trabalho, estudar o quadro hematológico de bubalinos, criados com e sem sombra na Amazônia Oriental.

\section{MATERIAL E MÉTODOS}

O experimento foi conduzido na Unidade de Pesquisa

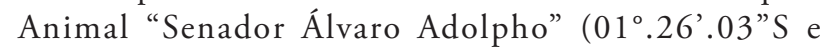
48․26'.03”W), da Embrapa Amazônia Oriental, Belém, Pará, Brasil, de janeiro a dezembro de 2009. O tipo climático é Afi (Köppen), com precipitaçáo pluvial média de 3.001,3 $\mathrm{mm}$ ano $^{-1}$, bem distribuída ao longo dos meses, com período mais chuvoso de janeiro a junho e menos chuvoso, de julho a dezembro. A temperatura média anual é de $27^{\circ} \mathrm{C}$, com média de umidade relativa do ar em torno de $85 \%$ e insolação anual de 2.400 horas ano-1 (Bastos et al. 2002). O solo dessa área é Latossolo Amarelo, fase pedregosa I, de textura argilosa.

Foram utilizadas 20 búfalas Murrah, entre quatro e cinco anos de idade, peso médio de 479,6 kg, cíclicas, não-gestantes e não-lactantes, clinicamente saudáveis, e distribuídas, aleatoriamente, em dois grupos (grupo CS com sombra e grupo SS - sem sombra). O grupo CS ( $\mathrm{n}=$ 10) permaneceu em piquetes de sistema silvipastoril e pastejo rotacionado intensivo, em Brachiaria humidicola, com sombra da leguminosa Racosperma mangium, plantadas com quatro metros de espaçamento, nas cercas perimetrais e divisórias, eletrificadas, com dois fios de arame liso (Moura Carvalho et al. 2001). O grupo SS $(\mathrm{n}=10)$ foi mantido em piquetes com o mesmo manejo alimentar, sem acesso à sombra. Os dois grupos tinham acesso à água para beber e sal mineral ad libitum e passaram por período de adaptação de 14 dias. As prerrogativas sanitárias, no tocante a vacinaçóes e controle de parasitoses, seguiram a indicação de Láu (1999).

Durante o período experimental foram registrados os dados climatológicos com auxílio do "Medidor de Stress Térmico" (marca Instruterm, modelo TGD-300, São Paulo, Brasil) que foi instalado no microclima apresentado em cada piquete de cada tratamento experimental (grupo CS e grupo SS). Foram registrados dados de temperatura do ar (máxima, média e mínima), umidade relativa do ar, temperatura de ponto de orvalho e temperatura de globo negro. As leituras das variáveis ambientais foram realizadas de $12 \mathrm{~h}$ às $13 \mathrm{~h}$ horas, duas vezes por semana, onde os dados eram registrados em datalogger. A partir dos valores de variáveis ambientais, foi calculado o índice ambiental: Índice de Temperatura de Globo e Umidade (ITGU), proposto por Buffington et al. (1981), determinado pela fórmula: $\mathrm{ITGU}=\mathrm{Tgn}+0,36 \mathrm{Tpo}+41,5$, onde, $\mathrm{Tgn}=$ Temperatura de Globo Negro ao Sol $\left({ }^{\circ} \mathrm{C}\right)$ e Tpo $=$ Temperatura do Ponto de Orvalho $\left({ }^{\circ} \mathrm{C}\right)$.

As coletas de sangue para determinar o eritrograma e número total de leucócitos foram realizadas a cada 14 dias, no horário de pico de temperatura ambiente local $(13 \mathrm{~h})$, pela punçáo da veia jugular externa, em tubos à vácuo, com anticoagulante etilenodi-aminotetracético sal dissódico (EDTA), a 5\%, obtendo-se três $\mathrm{ml}$ de sangue. Essas amostras foram levadas ao Laboratório de Análises Clínicas da Universidade Federal Rural da Amazônia (UFRA), para processamento, de acordo com a metodologia de Birgel (1982), com contagem celular manual.

Os dados das variáveis hematológicas foram expressos em média e desvio-padrão. $\mathrm{Na}$ análise dos dados climáticos do microclima dos tratamentos foi observada uma fase de transição, entre os períodos mais e menos chuvoso do ano, principalmente à tarde, em maio, junho e julho, com diminuição significativa da umidade relativa do ar e sensível aumento da temperatura do ar. Dessa forma, os dados foram organizados em três períodos: mais chuvoso (janeiro a abril), de transição (maio a julho) e menos chuvoso (agosto a dezembro).

O delineamento experimental foi inteiramente casualizado, em esquema fatorial de dois (tratamento) vs. três (período do ano). Foi usado o programa estatístico NTIA, versão 4.2.2 
(Embrapa 1997) e feitas análises de variância (ANOVA), para verificar o efeito dos tratamentos, períodos do ano e interação tratamento vs. períodos do ano, nas variáveis hematológicas. As médias foram comparadas pelo teste de Tukey a 5\% de probabilidade e feitas correlaçóes simples de Pearson para verificar a magnitude e direçáo da proporcionalidade das variáveis ambientais e hematológicas, observando-se a independência das variâncias dos pares de observaçôes utilizados.

\section{RESULTADOS E DISCUSSÃO}

Valores de ITGU de até 74 definem situaçáo de conforto, de 74 a 78, de alerta, de 79 a 84 , perigosa, e acima de 84, emergência (Baêta 1985). No presente trabalho, os valores desse índice demonstram condição térmica acima da considerada de conforto (ITGU $=74)$ e diferenças significativas entre os períodos do ano, com maiores valores na época menos chuvosa. Isso ocorreu, provavelmente, devido à ocorrência de elevada temperatura do ar, o que proporcionou condiçóes estressantes aos animais. Também, o ITGU sofreu influência significativa $(\mathrm{P}<0,05)$ dos tratamentos (Tabelas 1) e o grupo $S S$ apresentou valores mais elevados, o que, mais uma vez, confirma a importância do uso da árvore em sistemas de manejo, para proporcionar maior conforto térmico aos animais e melhorar o seu desempenho produtivo (Lourenço Júnior et al. 2002).

Os valores médios e máximos de hemácias (Tabela 2) estâo próximos dos citados por Silva (1986), Jain (2000) e Ciaramella et al. (2005), de $6,03 \times 10^{6} \mathrm{~mm}^{-3}, 6,34 \times 10^{6} \mathrm{~mL}^{-1}$ e $6,82 \times 10^{12} \mathrm{~L}^{-1}$, respectivamente. $\mathrm{O}$ número de hemácias sofreu influência dos tratamentos $(\mathrm{P}<0,05)$ e, no período

Tabela 1 - Índice de temperatura de globo e umidade (ITGU), ao sol e à sombra, em Belém, Pará.

\begin{tabular}{lcc}
\hline \multirow{2}{*}{ Tratamento } & \multicolumn{2}{c}{ Índice de Temperatura de Globo e Umidade } \\
\cline { 2 - 3 } & Máxima & Média \\
\hline Grupo SS & 92,0 & $89,1 \pm 2,8^{\mathrm{a}}$ \\
Grupo CS & 87,6 & $84,7 \pm 2,2^{\mathrm{b}}$ \\
\hline
\end{tabular}

a,b Médias dos ITGU nos tratamentos, seguidas de letras minúsculas distintas, na mesma coluna são diferentes $(P<0,05)$.

Tabela 2 - Número de hemácias $\left(\times 10^{6} \mathrm{~mm}^{-3}\right)$ em búfalas criadas ao sol (grupo SS) e à sombra (grupo CS), em Belém, Pará.

\begin{tabular}{|c|c|c|c|c|}
\hline \multirow{3}{*}{ Período } & \multicolumn{4}{|c|}{ Tratamento } \\
\hline & \multicolumn{2}{|c|}{ Grupo SS } & \multicolumn{2}{|c|}{ Grupo CS } \\
\hline & Máxima & Média & Máxima & Média \\
\hline Mais chuvoso & 10,0 & $7,2 \pm 1,3$ aA & 9,8 & $7,8 \pm 1,2^{\text {aA }}$ \\
\hline Transição & 7,9 & $7,2 \pm 0,5^{\mathrm{aA}}$ & 8,2 & $7,0 \pm 0,9 \mathrm{bA}$ \\
\hline Menos chuvoso & 9,1 & $7,9 \pm 0,7$ aA & 8,3 & $6,7 \pm 1,1^{\mathrm{bB}}$ \\
\hline
\end{tabular}

menos chuvoso do ano, o grupo SS apresentou valores mais elevados. Esse fato pode ser explicado pela elevaçáo da temperatura do ar, que interferiu nesse componente sanguíneo dos animais sem acesso à sombra, devido à hemoconcentração, em funçấo da perda de água pela evaporação (Silva et al. 2003). Esses resultados corroboram com os obtidos por Barcelos et al. (1989), que encontraram níveis mais elevados de hemácias, em temperatura ambiente elevada, e concluíram que os búfalos perdem mais calor, através da sudação, o que ocasiona hemoconcentraçẫo, embora Vieira (1995) não tenha observado diferenças estatísticas nas hemácias, entre grupos Controle e Estressado $\left(7,42 \times 10^{6} \mathrm{~mm}^{-3}\right.$ vs. $7,68 \times 10^{6} \mathrm{~mm}^{-3}$, respectivamente).

No período mais chuvoso do ano, o grupo CS teve maiores valores de hemácias, provavelmente pela elevada umidade relativa do ar, nesse período, que pode ter causado desconforto térmico e dificuldade em eliminar calor por evaporação. De acordo com Pereira (2005), elevada UR, aliada a temperatura do ar elevada, pode ser prejudicial ao animal, no tocante à perda de calor, por evaporaçáo. Nos períodos de transiçáo e menos chuvoso, conforme a umidade relativa do ar foi diminuindo, os animais deste grupo (CS) obtiveram valores menores de hemácias, enquanto no outro grupo não houve diferença significativa entre períodos do ano.

Em ambos os tratamentos, o número de leucócitos está acima dos descritos por Silva (1986), Jain (2000) e Ciaramella et al. (2005), de $8.200 \mathrm{~mm}^{-3}, 9.676 \mathrm{~mm}^{-3}$ e $7.220 \mathrm{~mm}^{-3}$ leucócitos, respectivamente (Tabela 3).

No período mais chuvoso, o grupo CS apresentou maiores valores de número de leucócitos que o grupo SS. De acordo com Ferreira Neto et al. (1978), o número de leucócitos é maior quando os animais estáo submetidos a estresse térmico. Assim, o menor número de leucócitos dos animais do grupo SS se deve, provavelmente, ao maior conforto térmico que esses animais obtiveram devido a um comportamento observado, durante o experimento, nesse período do ano, quando a elevação da precipitação pluvial favoreceu a formação de poças d'água na pastagem pelos búfalos desse grupo, uma

Tabela 3 - Número de leucócitos $\mathrm{mm}^{-3}$ em búfalas, criadas ao sol (grupo SS) e à sombra (grupo CS), em Belém, Pará.

\begin{tabular}{|c|c|c|c|c|}
\hline \multirow{3}{*}{ Período } & \multicolumn{4}{|c|}{ Tratamento } \\
\hline & \multicolumn{2}{|r|}{ Grupo SS } & \multicolumn{2}{|r|}{ Grupo CS } \\
\hline & Máxima & Média & Máxima & Média \\
\hline $\begin{array}{l}\text { Mais } \\
\text { chuvoso }\end{array}$ & 22.738 & $17.621 \pm 3.499 \mathrm{cB}$ & 32.356 & $19.661 \pm 6.290$ \\
\hline Transição & 22.323 & $18.278 \pm 3.379 \mathrm{bA}$ & 22.621 & $15.351 \pm 4.487$ св \\
\hline $\begin{array}{l}\text { Menos } \\
\text { chuvoso }\end{array}$ & 29.967 & $20.743 \pm 5.633^{\mathrm{aA}}$ & 26.456 & $18.546 \pm 4.864$ вв \\
\hline
\end{tabular}


vez que não tinham acesso à sombra, e onde se deitavam para dissipaçáo de calor e, consequente termorregulação. Esse resultado pode ser devido à maior eficiência de perda de calor por condução na água.

Por outro lado, nos períodos de transição e menos chuvoso, no grupo SS os valores de leucócitos foram mais altos, fato que pode ser explicado pela influência da temperatura mais elevada. Os resultados de Ferreira Neto et al. (1978) corroboram com os do presente estudo. Estes autores afirmam que em situaçóes de estresse térmico, em virtude da secreção endógena de corticosteróides, o número de leucócitos totais pode estar aumentado (leucocitose). Entretanto, para Shafie (2000), a contagem total de leucócitos, nos búfalos, não responde às circunstâncias climáticas sazonais.

Para bubalinos, os valores de referência do teor de hemoglobina são de $11,1 \mathrm{~g} \mathrm{dL}^{-1}$ (Jain 2000), 13,5 $\mathrm{g} \mathrm{dL}^{-1}$ (Ciaramella et al. 2005) e 11,37 g dL (Terzano et al. 2005). Segundo Jain (1993), vários fatores devem ser considerados, tais como raça, espécie, idade, sexo, hora do dia e estado fisiológico, uma vez que podem interferir nos valores de referência.

Não houve diferenças significativas dos valores de hemoglobina $(P>0,05)$ entre os tratamentos, nos três períodos do ano (Tabela 4). Esses resultados são semelhantes aos observados por Vieira (1995), em novilhas bubalinas submetidas a estresse térmico, onde não foram observadas diferenças entre Controle e Estressado (12,81 vs. 13,02 g dL${ }^{1}$, respectivamente). Por outro lado, o teor de hemoglobina sofreu efeito dos períodos do ano, onde níveis mais elevados $(\mathrm{P}<0,05)$ ocorreram nos períodos de transição e menos chuvoso, em ambos os tratamentos, provavelmente devido à influência da temperatura mais elevada. De acordo com Schmidt-Nielsen (1996), a hemoglobina é responsável pelo transporte de oxigênio dos pulmôes para os diferentes tecidos, e durante situação de estresse, a liberação do oxigênio se dá de forma mais rápida, o que contribui para o aumento da sua taxa de consumo, e consequentemente, elevação desse componente sanguíneo.

Tabela 4 - Valores de hemoglobina ( $\mathrm{g} \mathrm{dL}^{-1}$ ) em búfalas criadas ao sol (grupo SS) e à sombra (grupo CS), em Belém, Pará.

\begin{tabular}{|c|c|c|c|c|}
\hline \multirow{3}{*}{ Período } & \multicolumn{4}{|c|}{ Tratamento } \\
\hline & \multicolumn{2}{|c|}{ Grupo Sem Sombra (Grupo SS) } & \multicolumn{2}{|c|}{ Grupo Com Sombra (CS) } \\
\hline & Máxima & Média & Máxima & Média \\
\hline $\begin{array}{l}\text { Mais } \\
\text { chuvoso }\end{array}$ & 13,7 & $11,8 \pm 1,1 \mathrm{bA}$ & 14,4 & $12,1 \pm 1,7 \mathrm{bA}$ \\
\hline Transição & 15,6 & $14,0 \pm 0,9 \mathrm{aA}$ & 15,3 & $13,3 \pm 1,5 \mathrm{aA}$ \\
\hline $\begin{array}{l}\text { Menos } \\
\text { chuvoso }\end{array}$ & 14,8 & $13,8 \pm 0,7 a A$ & 16,4 & $13,6 \pm 1,7 \mathrm{aA}$ \\
\hline
\end{tabular}

a,b Médias dos tratamentos, dentro de cada período, seguidas de letras minúsculas distintas, na mesma coluna são diferentes $(P<0,05)$. $^{A B}$ Médias dos períodos, dentro de cada tratamento, seguidas de letras maiúsculas distintas, na mesma linha são diferentes $(P<0,05)$
Barcelos et al. (1989) encontraram níveis mais elevados no teor de hemoglobina em búfalos submetidos à temperatura ambiente elevada. Entretanto, segundo Shafie (2000), em bubalinos, o estresse térmico provocado pela exposição à radiaçấo solar direta causa diminuição na taxa de hemoglobina.

No presente trabalho constatou-se que os valores de volume globular oscilaram próximos daqueles descritos na literatura, de 31\% (Jain 2000), 34\% (Ciaramella et al. 2005) e $41 \%$ (Terzano et al. 2005). O volume globular não sofreu influência dos tratamentos e períodos do ano, nem da sua interação. Esse resultado corrobora com o obtido por Vieira (1995) que não observou diferenças entre os grupos Controle e Estressado (37,59 vs. 38,09\%, respectivamente), em novilhas bubalinas submetidas a estresse térmico. Na Paraíba, segundo Souza et al. (2007), os menores valores foram encontrados na estaçâo seca. De acordo com Silva et al. (2003), o estresse pelo calor de longa duraçáo pode diminuir o volume globular de bovinos, levando a uma hemoconcentração, em função da perda de água pela evaporaçáo, enquanto Barcelos et al. (1989) encontraram níveis mais elevados em búfalos submetidos à temperatura ambiente elevada.

Os leucócitos, hemácias e volume globular não se correlacionaram com a TA, UR e ITGU (Tabela 5). Esses resultados discordam dos encontrados por Shafie (2000), que encontraram correlação negativa entre concentração de hemoglobina e temperatura do ar. Segundo este autor, em bubalinos e bovinos, o estresse térmico provocado por exposição à radiação solar direta causa diminuiçáo nesta taxa. Entretanto, os búfalos apresentaram valores de hemoglobina superiores aos dos bovinos. A hemoglobina teve correlação significativa e negativa com a UR $(\mathrm{P}<0,05)$, e demonstra que, quando a variável UR se eleva, o valor da hemoglobina diminui.

Tabela 5 - Correlação entre variáveis hematológicas das búfalas experimentais da raça Murrah com variáveis climáticas em Belém, Pará.

\begin{tabular}{lccc}
\hline Variável & TA & UR & ITGU \\
\hline Leucócitos & $0,36^{\text {ns }}$ & $-0,39^{\text {ns }}$ & $0,33^{\text {ns }}$ \\
Hemácias & $0,14^{\text {ns }}$ & $-0,02^{\text {ns }}$ & $0,27^{\text {ns }}$ \\
Hemoglobina & $0,36^{\text {ns }}$ & $-0,57^{\star \star}$ & $0,23^{\text {ns }}$ \\
Volume Globular Médio & $0,21^{\text {ns }}$ & $0,033^{\text {ns }}$ & $0,27^{\text {ns }}$ \\
\hline
\end{tabular}

TA - Temperatura do ar; UR - Umidade relativa do ar; ITGU - Índice de temperatura de globo e umidade. Ns - não significativo; ${ }^{\star \star}$ - significativo à $1 \%$.

\section{CONCLUSÕES}

Independente do período do ano, as búfalas Murrah estấo sujeitas a ambiente hostil, sendo o período menos chuvoso o mais propício a estresse térmico, com os maiores valores de ITGU. Neste período, os teores de hemoglobina dos grupos com e sem sombra encontraram-se aumentados, provavelmente devido às condiçôes climáticas de elevadas temperaturas do ar. Por outro lado, considerando-se o 
número de hemácias e leucócitos dos animais com acesso à sombra, o período mais chuvoso do ano foi o mais prejudicial, provavelmente em função da umidade relativa do ar elevada, o que dificulta a liberação do calor dos animais, por evaporação, levando-os à condição de estresse térmico.

\section{AGRADECIMENTOS}

À Superintendência de Desenvolvimento da Amazônia - SUDAM, à Embrapa Amazônia Oriental, à Universidade Federal Rural da Amazônia - UFRA e à Coordenação de Aperfeiçoamento de Pessoal de Nível Superior - CAPES pela disponibilidade de recursos necessários para a execução do experimento.

\section{BIBLIOGRAFIA CITADA}

Baêta, F.C. 1985. Responses of lactating dairy cows to the combined effects of tempertature, humidity and wind velocity in the warm season. Tese de Doutorado, Agricultural Engeneering Department, University of Missouri, Columbia. 218 pp.

Barcelos, A.F.; Garcia, J.A.; Cardoso, R.M. 1989. Physiological reactions of buffaloes, zebus, taurin and mestizos under climate effect and diet. II - Sanguineous components. Revista da Sociedade Brasileira de Zootecnia, 12: $42-47$ (in Portuguese, with abstract in English).

Bastos, T.X.; Pacheco, N.A.; Nechet, D.; Sá, T.D.A. 2002. Climatic aspects of Belém in the last hundred years. Belém: Embrapa Amazônia Oriental. 31 pp. (Embrapa Amazônia Oriental. Documentos, 128) (in Portuguese).

Birgel, E.H. 1982. Hematology techniques of current use in clinical pathology clinic veterinary. Patologia Clínica Veterinária, Sociedade Paulista Veterinária, 7-23 (in Portuguese).

Birgel Júnior, E.H.; D’Angelino, J.L.; Benesi, F.J.; Birgel, E.H. 2001. Reference values of the erythrogram of Jersey breed, raised in São Paulo State. Arquivo Brasileiro de Medicina Veterinária e Zootecnia, 53: 164-171 (in Portuguese, with abstract in English).

Buffington, D.E.; Colazzo-Arocho, A.; Caton, G.H. 1981. Black globe humidity comfort index (BGHI) as comfort equation for dairy cows. Transaction of the American Society Agricultural Engineering, 4(24): 711-714.

Ciaramella, M.; Corona, M.; Ambrosio, R.; Consalvo, F.; Persechino, A. 2005. Hematological profile on non-lactating Mediterranean buffaloes (Bubalus bubalis) ranging in age from 24 months to 14 years. Research in Veterinary Science, 79: 77-80.

Embrapa. Empresa Nacional de Pesquisa Tecnológica em Informática para a Agricultura (Campinas-SP). 1997. Software NTIA. Versão 4.2.2: manual do usuário - ferramental estatístico. Campinas. 258 pp (in Portuguese).

Ferreira Neto, J.M.; Viana, E.S.; Magalhães, L.M. 1978. Veterinary Clinic Patology. Sl: Rabelo e Brasil. 293 pp (in Portuguese).

Jain, N.C. 1993. Essentials of Veterinary Hematology. Philadelphia: Lea e Febiger. 417 pp.

Jain, N.C. 2000. Scham's Veterinary Haematology. $5^{\circ}$ Ed. Philadelphia. Ed. Lea e Febiger, p. 1085-1088
Láu, H.D. 1999. Desease in buffalos on Brazil: diagnosis, epidemiology and control. 1. ed. Brasília: Embrapa. 202 pp (in Portuguese).

Lourenço Júnior, J.B.; Teixeira Neto, J.F.; Costa, N.A.; Baena, A.R.C.; Moura Carvalho, L.O.D. 2002. Alternative systems for feeding buffaloes in Amazon Region. In: 1st Buffalo Symposium of the Americas, Belém. Proceedings, p. 31-42.

Lourenço Júnior, J.B.; Castro, A.C.; Dantas, J.A.S.; Santos, N.F.A.; Alves, O.S.; Monteiro, E.M.M. 2006. Effect of the climatic variable on the physiology of buffaloes created in silvipastoril system, Belém, Pará State. In: IV Congresso Brasileiro de Biometeorologia - Mudanças Climáticas: Impacto Sobre Homem, Plantas e Animais, Ribeirão Preto/SP. Proceedings. São Paulo (in Portuguese). Resumos em CD room

Moura Carvalho, L.O.D.; Costa, N.A.; Lourenço Júnior, J.B.; Baena, A.R.C. 2001. It Surrounds electric for containment of animals. Belém: Emater. 22 pp (in Portuguese).

Paes, P.R.; Barioni, G.; Fonteque, J.R. 2000. Comparison of hematological values among different aged female goats of the Parda Alpina breed. Veterinária Notícias, Uberlândia, 1(6): 43-49 (in Portuguese, with abstract in English).

Pereira, J.C.C. 2005. Applied beddings of bioclimatology to the animal production. Belo Horizonte: FEPMVZ. 195 pp (in Portuguese).

Schmidt-Nielsen, K. 1996. Animal Fisiology - Environment Adaptation. 5.ed. São Paulo: Santos. 546 pp (in Portuguese).

Shafie, M.M. 2000. Physiology responses and adaptation of water buffalo, p. 2. In: Yousef, M.K. Stress physiology in livestock. 260 pp.

Silva, M.B. 1986. Contribution to the eritrocitário profile of buffaloes (Bubalus bubalis, Linnaeus, 1758) female of the Jafarabadi Murrah and Mediterranea races, created in the São Paulo State. Dissertação de Mestrado, Universidade de São Paulo, São Paulo. 115 pp (in Portuguese).

Silva, R.M.N.; Souza, B.B.; Arcoverde, M.C.P.; Tavares, G.P.; Marinho, M.L.; Benício, T.M.A. 2003. Effect of the period of the year on the hematology parameters of Sindi bovines in HalfBarren. In: Reunião Anual da Sociedade Brasileira de Zootecnia, 40, Santa Maria. Proceedings. Santa Maria: SBZ. CD Rom.

Souza, B.B.; Silva, R.M.N.; Marinho, M.L.; Silva, G.A.; Silva, E.M.N.; Souza, A.P. 2007. Physiological parameters and index of tolerance to the heat of bovines of the Sindi race in the halfbarren of Paraiba State. Ciências Agrotécnicas, Lavras, 3(31): 883-888 (in Portuguese, with abstract in English).

Terzano, G.M.; Allegrini, S.; Borghese. A.; Roncoroni, C., Alfieri, L. 2005. Metabolic and hormonal parameters in buffaloes, p. 219-248. In: Buffalo Production and Research, Ed. A. Borghese, FAO, REU Technical Series.

Vieira, R.J. 1995. Physiological and hormonal answers of buffaloes heifers submitted to thermal stress. Tese de Doutorado, Faculdade de Medicina Veterinária e Zootecnia/Universidade Estadual Paulista "Júlio de Mesquita Filho", Botucatu. 148 pp. (in Portuguese).

Recebido em 09/05/2010

Aceito em 24/11/2010 